\title{
Estrategias de integración electoral multinivel: presidentes e intendentes peronistas en el conurbano bonaerense (2005-2015)*
}

Multilevel electoral integration strategies: peronist presidents

and mayors in Buenos Aires'suburbs.

\section{ANDRÉS LACHER **}

Consejo Nacional de Investigaciones Científicas y Técnicas, Argentina.

Instituto de Investigaciones Jurídicas y Sociales «Ambrosio L. Gioja», Facultad de Derecho, Universidad de Buenos Aires, argentina

lacherandres@gmail.com

El carácter descentralizado que presenta la integración electoral multinivel en partidos estratárquicos que compiten en distintas arenas, se torna evidente en las largas y complejas negociaciones pre-electorales que se desarrollan entre quienes desempeñan cargos ejecutivos. Como resultado de ello, los oficialismos nacionales, provinciales y municipales pueden adoptar estrategias de coordinación o competición que impactan sobre las arenas de sus contrapartes. Este artículo explica la variación de este tipo de estrategias entre presidentes e intendentes peronistas de los 24 partidos del conurbano bonaerense, a partir del análisis de resultados electorales y estrategias de integración correspondientes a los comicios anteriores. Para ello, se conceptualiza a la vinculación entre oficialismos de distintos niveles como una relación de principales y agentes, al tiempo que se recurre a enfoques sobre la cooperación. Este marco analítico da lugar a dos hipótesis, cuyas consecuencias observacionales son testeadas a partir de regresiones logísticas y paneles de efectos fijos.

\section{Introducción}

Los candidatos de los partidos políticos que compiten en sistemas federales requieren de la cooperación de otros actores de su partido que contienden en la arena municipal, provincial o nacional, con el objetivo de obtener resultados electorales que les permitan acceder o permanecer en posiciones de gobierno (Avelino et. al, 2013; Samuels, 2003). Si bien en determinados

\footnotetext{
* Articulo aceptado para su publicación el 12 de diciembre de 2018. Este artículo constituye una síntesis de la tesis de maestría del autor (Lacher, 2018).

** El autor agradece los aportes de dos evaluadores anónimos de Revista SAAP a una versión previa de este artículo.
} 
contextos donde priman principios organizativos jerárquicos (Carty, 2004) esta cooperación no presenta mayores inconvenientes, llegando incluso a darse por descontada por los actores políticos, en otros escenarios, este tipo de desenlaces no pueden ser garantizados a priori. Este es el caso de los partidos donde la integración electoral entre los dirigentes que compiten en diferentes niveles presenta un carácter descentralizado (Leiras, 2006), situación que se suele evidenciar en modelos organizativos que responden a imperativos estratárquicos (Carty, 2004). En estos entornos, la eventual cooperación entre incumbents de distintos niveles de competencia suele quedar supeditada a la concreción de negociaciones, y sus resultados impactan o bien en la cantidad de cantidatos, o bien en las etiquetas mediante las cuales éstos compiten en las elecciones.

Esta última dinámica de integración multinivel ha sido señalada como una característica del peronismo argentino (Scherlis, 2013). Es por ello que este trabajo se centra en los resultados de la integración multinivel entre un conjunto de actores preponderantes al interior de esa fuerza política ${ }^{1}$, como es el caso presidentes e intendentes en los veinticuatro partidos del conurbano bonaerense $^{2}$ durante el periodo $2005-2015^{3}$, en tanto las características que detentaron estos distritos y sus respectivos intendentes (Ollier, 2010) habilitaron la concreción de procesos de negociación directa en la integración entre los niveles de competencia municipal y nacional ${ }^{4}$ (Szwarcberg Daby, 2016).

En dichos procesos se presentaron tanto situaciones de cooperación, como de competición entre oficialismos de ambos niveles. La primera de

1 El estudio se circunscribe al peronismo en tanto se registró en su interior una variación de nuestra variable dependiente de interés. En este sentido, se entiende por «peronistas» tanto a candidatos oficialistas para cargos municipales y nacionales que compitieron por el Frente para la Victoria (FPV) como a determinados intendentes que contendieron en las boletas de Hilda Duhalde en 2005, Eduardo Duhalde en 2011 y Sergio Massa en 2013 y 2015. El resto de los postulantes a cargos nacionales del peronismo no ha sido tenido en cuenta en virtud de que ningún intendente del conurbano (ni sus candidatos al Concejo Deliberante) compitieron en su respectiva boleta.

2 Los partidos son: La Matanza, Lomas de Zamora, Quilmes, Almirante Brown, Merlo, Moreno, Lanús, Florencio Varela, General San Martín, San Miguel, Tigre, Tres de Febrero, Avellaneda, Malvinas Argentinas, Berazategui, Morón, Esteban Echeverría, Vicente López, José C. Paz, Hurlingham, Ituzaingó, Ezeiza, San Fernando y San Isidro.

3 El periodo estudiado se justifica por la semejanza entre la filiación política de los dos presidentes y la mayoría de los intendentes del conurbano durante toda la década. Cabe señalar que el análisis no pudo ser extendido a las elecciones de 2003 por carecer de datos comparables respecto a la elección previa.

$4 \quad$ En este punto se suscribe a lo señalado por Szwarcberg Daby, quien sostiene que las características de los intendentes del conurbano los habilitan para «(...) negociar su futuro político con las autoridades nacionales soslayando a sus dirigentes provinciales» (Szwarcberg Daby, 2016: p. 579), razón por la cual no se contemplará a estos actores en el análisis propuesto, aun cuando sus cargos se dirimieron en elecciones concurrentes junto con los cargos municipales y nacionales. 
ellas se evidenció en los casos donde intendentes o presidentes apoyaron al incumbent de otra arena, absteniéndose de patrocinar a sus contendientes peronistas. La segunda se dio en los escenarios donde los oficialismos nacionales patrocinaron a otras «listas colectoras», de «adhesión» u otras listas internas dentro del FPV, o bien en los casos donde los (candidatos de los) intendentes del conurbano, compitieron en representación de otras expresiones nacionales del peronismo. Como las estrategias de cooperación y competición fueron implementadas tanto por oficialismos municipales como nacionales, denominaremos a las que se produjeron desde los actores distritales hacia los nacionales como integración «ascendente», mientras que las que operaron en sentido inverso serán denominadas como «descendente».

A partir de la variación señalada, el objetivo de este trabajo es explicar por qué los dirigentes que ocupan cargos ejecutivos a nivel nacional y municipal optan por coordinar sus estrategias electorales con los oficialismos de otras arenas de competencia que pertenecen a su partido, o deciden apoyar a los contendientes de estos últimos. En concreto, se busca responder al siguiente interrogante: ¿Qué explica la adopción de estrategias de integración divergentes por parte de los dirigentes de un partido político que ocupan cargos ejecutivos, en los procesos de integración electoral multinivel?

Interrogantes como el presentado han sido abordados por distintos enfoques, entre los que se encuentran los centrados en la desnacionalización, desinstitucionalización y federalización que presenta el sistema de partidos argentino (Calvo y Escolar, 2005; Leiras, 2006) y su impacto en el grado de congruencia que presentan las coaliciones multinivel (Clerici, 2015; Mauro, 2018); los (des)incentivos de integración vertical que exhibe el régimen electoral en determinadas arenas subnacionales (Borges y Lloyd, 2016; Clerici, 2014); y el patrocinio de los ejecutivos nacionales y provinciales a listas peronistas «disidentes» (Galván, 2011, 2011b).

Sin embargo, los argumentos esgrimidos por estas aproximaciones distan de ser concluyentes para el caso que se analiza. Esto se debe, en primer lugar, a que las contribuciones enfocadas en los sistemas de partido y en las coaliciones partidarias, poseen una unidad de análisis diferente que impide extender sus conclusiones hacia las estrategias de los incumbents al interior de un partido político. En segundo lugar, los argumentos centrados en los sistemas electorales no pueden dar cuenta de la existencia de estrategias divergentes en distritos donde rigen las mismas reglas electorales. En tercer lugar, la tesis del patrocinio de «listas disidentes» no contempla la capacidad de autonomía y acción estratégica de los oficialismos subnacionales, los cuales sólo tenderían a implementar tácticas de competición ascendente al momento de ser patrocinados por un actor político de mayor o igual relevancia. Por último, cabe señalar que la arena municipal es un terreno escasamente explorado por todos los enfoques señalados. 
Este trabajo pone énfasis en variables que han sido soslayadas por los aportes mencionados, como son los casos de los resultados electorales y las estrategias implementadas en los comicios previos. Para justificar su pertinencia se construye un marco analítico a partir de la teoría de juegos, que incluye el enfoque de principal-agente y algunos elementos relativos a la cooperación.

A partir del mismo, se formulan dos hipótesis. La primera de ellas postula que oficialismos municipales y nacionales sólo vuelven a delegar labores electorales en sus contrapartes cuando los resultados de los últimos comicios les ofrecen certidumbre. La segunda apunta a la incidencia del tipo de estrategia elegida por el oficialismo de otro nivel de competencia en los comicios anteriores, en la táctica implementada por intendentes y presidentes en la elección siguiente. Finalmente, también se testea la reciprocidad de las estrategias entre presidentes e intendentes, empleando a cada uno de los veinticuatro municipios estudiados como unidad de análisis.

Para concluir, los hallazgos de este trabajo se exponen en el último apartado, donde se aborda el formato de competición electoral del peronismo, junto con los insumos que presenta el marco analítico para el estudio de la cooperación intrapartidaria en contextos de competencia multinivel.

\section{El enfoque de agente-principal en la integración multinivel}

En partidos que deben afrontar competencias electorales multinivel y que construyen su vínculo con los votantes a partir de diversos mecanismos (Kitschelt, 2000), los dirigentes que ocupan cargos públicos a nivel nacional y municipal pueden beneficiarse de las potenciales ventajas que ofrece la integración con sus pares que compiten en otras arenas, delegando determinadas labores relativas a las campañas electorales en sus contrapartes. En este sentido, si se toma como ejemplo un partido que construye su vínculo con los votantes a partir de mecanismos clientelares y carismáticos, cabría esperar, por un lado, que los intendentes deleguen en sus candidatos nacionales la elaboración de diagnósticos y propuestas electorales que contribuyan a forjar el valor informativo de la etiqueta partidaria; sobre todo en contextos donde los «atajos» (Downs, 1957) que ésta proporciona dependen en gran medida de las características de los dirigentes que compiten en la arena nacional, al momento que estos se encuentran identificados con la imagen del partido, resultando figuras indicativas de su posición ideológica (Kim, 2015). Por otro lado, en estos casos se esperaría que los candidatos nacionales encomienden a los intendentes de su partido los aspectos relativos al trabajo territorial y la movilización de sectores del electorado. 
Este proceso de delegación mutua permite que la dinámica entre dirigentes que compiten en diferentes niveles pueda ser conceptualizada como una relación de agente-principal, vínculo que se caracteriza por la autorización del principal al agente, para que éste emprenda determinadas actividades con la expectativa de que las cumpla, de manera tal que los resultados obtenidos sean los deseados por el principal (Van Houten, 2009). Si se acepta lo anterior, resulta posible señalar que candidatos que compiten en la arena municipal ocupan el rol de principales en los procesos de integración ascendente, mientras que los postulantes nacionales desempeñan igual papel en lo que hemos definido como integración descendente. De esta manera, a diferencia de la utilización habitual del enfoque de agente-principal, en el modelo propuesto ambos actores (intendentes y presidentes) ocupan la posición de agentes y principales en forma simultánea en el marco de elecciones concurrentes.

Una primera ventaja de conceptualizar los procesos de integración multinivel a partir de este modelo analítico descansa en los insumos teóricos del mismo para caracterizar los potenciales riesgos que presentan los procesos de delegación señalados. Estos pueden originarse a partir del conflicto de intereses entre ambos actores o del ocultamiento de información y acciones por parte de los agentes (Van Houten, 2009), los cuales pueden conspirar contra la eficiencia de las tareas realizadas, sobre todo en contextos donde estos últimos carecen de incentivos para favorecer los objetivos del principal (Moe, 1984).

En el marco de los procesos de integración multinivel, el conflicto de intereses puede producirse en el momento en que los agentes privilegian el desempeño de la organización partidaria en su propia arena de competencia, en detrimento de los resultados obtenidos por el principal en otros niveles de gobierno. Esta situación puede agravarse en el marco de sistemas de partidos desnacionalizados y cuando las carreras políticas de los dirigentes transcurren en un solo nivel de competencia (Van Houten, 2009). A modo de ejemplo, este último escenario se produce cuando los dirigentes que ocupan cargos ejecutivos en la arena municipal no poseen ambiciones políticas verticales (Lodola, 2009), es decir, que no aspiran necesariamente a continuar su carrera política en cargos provinciales o nacionales.

El ocultamiento de información por parte del agente puede deberse a su posibilidad de obtener réditos a partir del manejo exclusivo de algunos aspectos relativos a su arena de competencia a expensas del interés del principal. Por su parte, el ocultamiento de acciones se puede producir cuando el principal no puede determinar con exactitud la influencia del desempeño del agente en los resultados que arrojan los procesos de integración multinivel. En este sentido, la escasa información de la que disponen los 
principales no permite, por sí sola, dar cuenta de cuánto pudo haber sido la contribución efectiva de los dirigentes de cada arena de competencia al resultado global, y mucho menos si los resultados obtenidos por el partido se deben al trabajo territorial de los actores distritales o al efecto arrastre de los que compiten la arena nacional. Cabe señalar que esta incertidumbre se vuelve aún mayor en el marco de elecciones concurrentes (Leiras, 2006).

Los potenciales problemas de agencia derivados del conflicto de intereses, ocultamiento de información o acciones no constituyen el desenlace necesario de todos los procesos de delegación en el marco de la integración multinivel, ni los mismos acarrean necesariamente la abdicación y la transferencia de autoridad por parte del principal hacia el agente (Lupia y McCubins, 2000). Esto se debe a que tanto dirigentes municipales como nacionales poseen mecanismos para monitorear el desempeño de sus agentes, cuestión que les permite premiarlos cuando consideran que estos han obrado en forma satisfactoria, volviendo a delegar en ellos labores proselitistas en los próximos comicios; o bien, encontrar mecanismos de control o sanción cuando no ha sido el caso, tales como la estimulación de la competencia entre agentes alternativos en otras arenas o simplemente, la determinación de no volver a delegar en los próximos comicios.

Para ello, los principales suelen recurrir a fuentes de información incompletas e imperfectas que les otorgan una idea aproximada de la distancia entre sus expectativas y la labor efectiva de los agentes en el proceso de integración multinivel. Debido a la relevancia que presentan para la carrera política de los actores involucrados y por los escasos costos que acarrea su obtención, los resultados electorales suelen constituir estos «proxies» (para utilizar una expresión propuesta por Leiras), empleados por los principales con el objetivo de monitorear a sus contrapartes que compiten en otras arenas.

En particular se partirá del supuesto de que los oficialismos operan bajo una lógica retrospectiva y los últimos resultados electorales se presentan como una de las informaciones más actualizadas, que les permite tomar determinaciones en relación a su integración con los agentes en la próxima elección.

De esta manera, dados incumbents retrospectivos de idéntico partido cuyos cargos correspondientes a distintas arenas se dirimen en forma concurrente y donde alguno de ellos posee la expectativa de desarrollar su carrera política en un mismo nivel de competencia, cabría esperar que estos adopten estrategias de cooperación (competición) en situaciones de (in)satisfacción con los resultados electorales que arrojó el último proceso de integración multinivel (hipótesis 1).

Acorde a lo planteado, las estrategias de coordinación se producirían en escenarios donde los últimos resultados electorales les ofrecen una cuota de 
certidumbre a los dirigentes sobre los potenciales beneficios de la delegación de tareas en sus agentes, lo cual redunda en la cooperación futura entre oficialismos de distintos niveles de competencia.

Por su parte, lo que hemos definido como estrategias competitivas, se presentarían en escenarios donde se debilitan los incentivos percibidos por los principales para participar en procesos de integración multinivel con los oficialismos de otras arenas, debido a la insatisfacción respecto al proceso de delegación de los últimos comicios, ya sea porque estos perciben que los agentes no han realizado con éxito la tarea encomendada o han escatimado esfuerzos para cumplimentar la misma. En estos casos los actores políticos con márgenes de autonomía que buscan permanecer en posiciones de gobierno poseen mayores incentivos para adoptar las estrategias de control, entre las que contemplaremos sólo la remoción o el estímulo a la competencia entre agentes alternativos en otras arenas.

Para testear esta hipótesis en los casos de integración ascendente, se partirá del supuesto de que los oficialismos municipales evalúan la magnitud del efecto arrastre que le puede proporcionar su integración con los candidatos nacionales, a partir de la valoración de la diferencia entre la cantidad de votos obtenidos por los candidatos para categorías municipales y nacionales al interior de sus respectivos distritos.

En este sentido, cabría esperar que los intendentes del conurbano implementen una estrategia de coordinación ascendente cuando el oficialismo nacional (o sus respectivos candidatos) han obtenido más votos que los postulantes municipales al interior de sus distritos en la última elección. En estos casos, se presume que los intendentes han experimentado las ventajas del efecto arrastre de los candidatos nacionales, razón por la cual querrían continuar integrando la boleta electoral junto con sus agentes y, de este modo, se abstendrían de apoyar a candidatos partidarios no oficialistas que aspiran a ocupar cargos nacionales en los próximos comicios.

Por su parte, los casos donde los oficialismos municipales obtienen al interior de sus distritos registros similares y hasta incluso mayores que los candidatos nacionales, es posible que los primeros duden de las ventajas de competir bajo la etiqueta partidaria de los segundos en el futuro. Esto puede desembocar en que en las elecciones siguientes los dirigentes locales no deseen movilizar sus estructuras electorales en favor del oficialismo nacional o que, insatisfechos con los réditos obtenidos y con incertidumbre sobre su futuro, decidan reemplazar a sus agentes a partir de la implementación de una estrategia de integración competitiva que se traduce en el apoyo a dirigentes que rivalizan con estos en las elecciones sucesivas.

Asimismo, desde la perspectiva de los candidatos nacionales, las situaciones en las que los registros obtenidos por los postulantes distritales supe- 
ran su propia proporción de votos, pueden constituir un elemento indicativo de que el intendente habría escatimado esfuerzo en favor de sus pares nacionales o que habría privilegiado su arena de competencia en detrimento de estos últimos. A la vez, esta situación, también contribuiría a que se abstengan de delegar el trabajo territorial en los próximos comicios.

A partir de lo señalado, la primera consecuencia observacional de la hipótesis presentada es que la integración con candidatos nacionales que obtuvieron el mayor (menor) caudal de votos al interior del distrito con respecto a los — candidatos de los_ intendentes en la elección anterior, incrementó la probabilidad de la adopción de una estrategia de coordinación (competición) ascendente en la siguiente elección.

Por su parte, los liderazgos nacionales en su rol de principales, se enfrentan con una serie de dificultades a la hora de cotejar la distancia entre la contribución efectiva y el aporte potencial que hubieran podido cosechar sus agentes municipales en el marco de los procesos electorales. Esto se debe a que la variación en las proporciones de votos obtenidas en las categorías nacionales en cada municipio no permiten por sí solas evaluar la efectividad en el desempeño de distintos actores distritales que compiten en contextos que varían en características socioeconómicas o que presentan niveles disímiles de fragmentación política, cuestiones que pueden impactar en el desempeño partidario a nivel local.

Con el objetivo de superar esta potencial limitación que presenta la evaluación directa de resultados electorales, se procederá como si los mismos fueran valorados por el oficialismo nacional a partir de la media de la proporción de votos de los distritos del conurbano, cuyos intendentes adoptaron una estrategia de integración cooperativa en la última elección. La misma nos permitirá ponderar el último resultado de las categorías nacionales al interior de los municipios, con el objetivo de explicar la variación en las estrategias posteriores de integración descendentes de los liderazgos nacionales.

De este modo, cabría esperar que los oficialismos nacionales adopten una estrategia de cooperación con los intendentes municipales en los distritos donde cosecharon desviaciones positivas respecto a la media de las proporciones de votos en las categorías nacionales en los últimos comicios. En estos escenarios los oficialismos de mayor rango tenderían a respaldar a sus agentes con mayor capacidad o disposición para movilizar votos en el pasado inmediato y se abstendrían de patrocinar a sus rivales distritales en la siguiente elección.

Por su parte, en los distritos donde las proporciones de votos en las categorías nacionales presentan resultados inferiores a la media, los dirigentes de mayor rango pueden estar insatisfechos con la labor de sus agentes al 
tener reparos acerca de su efectividad o disposición para captar votos a nivel territorial en el futuro. En este marco, se espería que aquellos fomenten la competición entre agentes alternativos, distribuyendo su apoyo entre el intendente y sus rivales distritales en la próxima elección.

En función de lo señalado, la segunda consecuencia observacional de la primera hipótesis es que en los municipios donde las categorías nacionales registraron desviaciones positivas (negativas) con respecto a la media obtenida en los distritos donde los intendentes adoptaron estrategias de cooperación en la elección anterior, aumentó la probabilidad de que los oficialismos nacionales adopten una estrategia de coordinación (competición) descendente en la siguiente elección.

\section{La integración multinivel en interacciones reiteradas: un enfoque sobre la cooperación}

Lejos de presentarse como inocuas, las estrategias competitivas tienen impacto en otros niveles de gobierno, ya que disminuyen las posibilidades de los incumbents de idénticos partidos de permanecer en sus cargos ejecutivos, al tiempo que podrían incrementar las probabilidades de fragmentación en sus respectivas legislaturas. Este tipo de consecuencias mediatas de las estrategias estudiadas justifican la necesidad de explorar la dimensión dinámica de los procesos de integración multinivel.

En este punto, el modelo tradicional de agente-principal presenta una limitación, en tanto aborda los problemas de delegación en interacciones estáticas de una vuelta (Moe, 1984), en forma similar a cómo los mismos han sido explorados hasta el momento. Si bien este constituye un buen punto de partida, resulta insuficiente para examinar contextos donde los procesos de delegación tienen posibilidades de reiterarse en el tiempo (Bendor et. al, 2001), tal como ocurre entre los oficialismos que integran partidos políticos que compiten en elecciones consecutivas en diferentes arenas.

En estos casos donde los incumbents no poseen certezas acerca de cuándo será su última interacción (Axelrod, 1984), las distintas estrategias de integración multinivel adoptadas por los principales en el presente pueden tener relevancia en «rondas» sucesivas. Esto se explica por las mencionadas consecuencias negativas (positivas) que acarrea en otra arena una estrategia competitiva (de cooperación) adoptada por un incumbent nacional o municipal, las cuales pueden inducir a sus pares a adoptar idénticas estrategias en las próximas elecciones, a modo de represalia (reciprocidad).

De esta manera, si se contempla la posibilidad de repetir las interacciones en el futuro, los oficialismos no sólo definen su estrategia de integración 
multinivel a partir de los resultados electorales obtenidos en el proceso de integración anterior — tal como hemos señalado en la sección precedente-, sino que también contemplan la estrategia implementada por la contraparte en los comicios anteriores ${ }^{5}$.

En caso de que las estrategias de presidentes e intendentes obedecieran (sólo) a las tácticas implementadas por los oficialismos de otros niveles de competencia y emularan de manera indefinida la estrategia adoptada por sus contrapartes en la elección anterior, las secuencias de integración multinivel obedecerían a una dinámica que en teoría de juegos se denomina tit for tat. La misma supone que los actores tienden a cooperar en la primera ronda del juego y luego, en las interacciones sucesivas, emulan la estrategia implementada por su contraparte en la ronda anterior (Axelrod, 1984).

De este modo, si a los supuestos de la primera hipótesis se le añade la certeza de los incumbents de volver a interactuar en elecciones sucesivas, cabría esperar que oficialismos de idéntico partido repliquen la estrategia de cooperación (competición) implementada por sus contrapartes en la elección anterior (hipótesis 2).

De operar bajo la lógica planteada en la hipótesis, las estrategias de cooperación multinivel implementadas por presidentes e intendentes tienen posibilidad de ser replicadas en las elecciones siguientes. Por contrapartida, cabría esperar que, ante el rechazo de ambos oficialismos a las tácticas competitivas implementadas por sus contrapartes en el pasado inmediato, estos repliquen dichas estrategias en el siguiente proceso electoral a modo de represalia.

A partir de estas premisas, es posible deducir dos consecuencias observacionales de la segunda hipótesis. De acuerdo con la primera de ellas, cabría esperar que los intendentes que adoptaron una estrategia de integración competitiva en la elección precedente y modificaron su estrategia por una de coordinación en la siguiente elección, poseyeron mayores probabilidades de afrontar estrategias competitivas descendentes por parte de los oficialismos nacionales en dichos comicios. Por su parte, la segunda consecuencia observacional es que los intendentes que afrontaron victoriosos las estrategias competitivas de los oficialismos nacionales en las elecciones previas tuvieron mayores probabilidades de replicar a sus contrapartes con estrategias competitivas ascendentes en los siguientes comicios.

Como en determinadas circunstancias los imperativos que se originan a partir de la evaluación de resultados electorales y la reciprocidad con estrategias precedentes pueden presentar incentivos de integración contradictorios, no puede señalarse $a$ priori que existan en todos los casos estrategias óptimas de integración multinivel para los oficialismos. 
Si se continúa con la pretensión de explicar la integración multinivel desde una perspectiva dinámica, resulta preciso destacar que las distintas estrategias adoptadas no sólo tienen impacto en la siguiente ronda, sino que también pueden tener relevancia en el futuro, conspirando o favoreciendo las posibilidades de cooperación interpartidaria en las elecciones subsiguientes. En este sentido, si en algún punto se produce esta desaprobación del desempeño de un incumbent que compite en otro nivel y las interacciones sucesivas siguen una dinámica (similar a la) de tit for tat, las consecuencias a largo plazo dan a lugar a la concreción de un equilibrio competitivo multinivel al interior de un distrito, el cual tiende a exacerbar la brecha entre las estrategias racionales a nivel individual y la situación colectivamente óptima (Bendor et. al, 2001) para el partido en una jurisdicción determinada. Es en estos casos donde los incentivos y los objetivos de los actores que ocupan cargos ejecutivos pueden optar por competir en representación de etiquetas alternativas, que por sus características, suelen cumplir los requisitos para ser denominadas como partidos en un sentido institucional-mínimo pero no en un sentido funcional (Kitschelt, 2000).

Por contrapartida, en los distritos en donde los actores que ocupan cargos ejecutivos en distintos niveles reproducen estrategias de coordinación multinivel adoptadas por sus contrapartes en elecciones anteriores, tienden a producirse equilibrios cooperativos multinivel, los cuales generan percepciones compartidas en torno a las ventajas colectivas e individuales de la continuidad de los procesos de delegación multinivel en el tiempo. En estos casos, los oficialismos suelen estimar los recursos que pueden obtener compitiendo por dentro del partido y, por ende, esperan desarrollar sus carreras políticas en su interior.

Si lo anterior es correcto, es posible deducir una última consecuencia observacional de la segunda hipótesis en torno a los distintos tipos de equilibrios en los municipios estudiados. En este sentido, cabría esperar una relación negativa entre la cantidad de ocasiones que los intendentes de un municipio implementaron estrategias de coordinación ascendentes y la mediana de competitividad descendente que estos afrontaron al interior de sus respectivos distritos.

\section{Metodología}

Este apartado tiene por objeto ofrecer una descripción de la operacionalización de las variables independientes y de resultado presentadas en las hipótesis, junto con la justificación de las variables de control y las características de los modelos que se presentan en la próxima sección. 
Acorde a lo planteado en la introducción, la variable dependiente del estudio es la estrategia de integración electoral multinivel adoptada por los oficialismos nacionales y municipales al interior de un partido político, la cual puede asumir los valores de cooperación o competición. Dadas las características del caso estudiado, en los procesos de integración ascendente, la variable asume valores dicotómicos, mientras que en la integración descendente presenta valores continuos. Esto se debe tanto a los resultados políticos como a las características de las reglas electorales adoptadas durante el periodo analizado, las cuales les permitieron a los candidatos nacionales patrocinar en forma simultánea a numerosos candidatos a nivel distrital, mientras que esta posibilidad no se concretó en el caso de los candidatos distritales, quienes únicamente pudieron apoyar a un sólo candidato de mayor rango.

En función de lo señalado, en los casos de integración ascendente la variable dependiente puede asumir dos valores: de cooperación o competitiva. La primera de ellas se produce cuando los intendentes se integran apoyando a los candidatos del oficialismo que compiten en otro nivel, mientras que la segunda se origina cuando el apoyo se dirige hacia los que rivalizan con aquellos. Dadas las características categóricas y dicotómicas de esta variable dependiente, se testea la primera consecuencia observable de la primera hipótesis junto con la segunda implicación de la segunda hipótesis a partir de una regresión logística.

Para construir la variable dependiente en los casos de integración descendente se empleó un indicador que se obtiene mediante un análisis de componentes principales de dos variables originales: el número efectivo (Laakso y Taagepera, 1979) de «listas internas» 0 «colectoras» del oficialismo nacional en el municipio y el margen de victoria obtenido por la lista que obtuvo el primer lugar en el distrito sobre su competidora inmediata (Clerici et. al, 2016). Este indicador posee la virtud de presentar medidas cotejables de los resultados registrados durante la vigencia de dos sistemas electorales diferentes, al tiempo que ofrece una síntesis de la información de componentes que presentan colinealidad entre sí (en tanto ambos explican la variación de nuestra variable dependiente) a partir de una transformación lineal que presenta la dimensión latente de los dos componentes arriba mencionados (Clerici et. al, 2016).

Más allá de las ventajas señaladas, el empleo de la competitividad como indicador de las estrategias de integración descendente posee una limitación, en tanto no permite distinguir los casos en que los incrementos en la competitividad a nivel municipal se originan a partir del patrocinio de incumbents o candidatos de otros niveles de competencia, o si la misma se gesta al interior del distrito en cuestión como resultado de estrategias de 
actores partidarios locales que no responden al ejecutivo municipal ni al nacional. Dicha limitación se evidencia en los resultados no significativos obtenidos en el cuarto modelo, donde este es empleado como variable de interés y en algunos outliers registrados en el Gráfico 1, utilizado en el testeo de la tercera consecuencia observacional de la segunda hipótesis.

Una vez realizada esta aclaración, es preciso señalar que, luego de la transformación de las dos variables originales, la medición de la competitividad presenta una media de cero y desviación estándar de uno. A partir de ello, los casos donde los registros que más se alejan del promedio en sentido negativo, es decir, las situaciones en que las colectoras o las listas de las primarias que no responden al intendente obtienen escaso apoyo electoral, son considerados como evidencia de estrategias de coordinación descendente. Por contrapartida, en las situaciones inversas, donde se incrementa la competitividad en los municipios del conurbano y se registran resultados positivos alejados de la media, se considera que se produjo una estrategia competitiva descendente. Cabe mencionar que en las 106 elecciones analizadas, esta variable presenta valores que van de un mínimo de -0.95 a un máximo de 5.70, con una desviación estándar de 1.28.

Dadas las características continuas de esta variable de resultado, y a partir del hecho de que las variables de interés y control fueron recolectadas en series de tiempo, pretendiendo observar su impacto al interior de distritos con características específicas no observadas que se presumen temporalmente constantes, se emplea un modelo de panel con efectos fijos con análisis robusto de los errores para testear la segunda implicación observable de la primera hipótesis y la primera consecuencia observable de la segunda conjetura.

La tercera consecuencia observacional de la segunda hipótesis se evalúa empleando a cada uno de los 24 distritos estudiados como unidad de análisis a partir de un diagrama de dispersión con una línea de regresión suavizada cuyos ejes se construyen a partir de las variables dependientes de los primeros cuatro modelos. El eje vertical se elabora a partir de la mediana de la competitividad descendente registrada al interior de cada municipio, mientras que el horizontal presenta la cantidad de ocasiones que los intendentes de cada municipio adoptaron estrategias de cooperación ascendentes a lo largo de las seis elecciones celebradas durante el periodo 2005-2015.

La variable independiente de interés del primer modelo, donde se busca explicar la incidencia del monitoreo de resultados electorales en las estrategias de integración ascendente, se obtiene a partir de la resta entre la proporción de votos registrada por (los candidatos de los) intendentes en la categoría municipal y la proporción de votos obtenida por los contendien- 
tes nacionales que ocuparon el primer lugar ${ }^{6}$ en la boleta con los que estos se han integrado en la última elección. El resultado de esta operación da a lugar a una variable continua que puede presentar valores negativos cuando la proporción de votos obtenida por el (los) candidato(s) nacional(es) supera la cosecha del (los) postulante(s) de la categoría municipal y registros positivos cuando se da la situación contraria. El segundo caso resulta indicativo de que los oficialismos municipales no han obtenido ventajas a partir de la integración multinivel o que han escatimado esfuerzos en favor de sus pares nacionales; mientras que en el primer caso ilustra la situación inversa, donde se espera que se incrementen las probabilidades de que los intendentes vuelvan a delegar labores electorales en el oficialismo nacional en los próximos comicios. En las 122 observaciones recolectadas esta variable presenta una media de -1.2 y desviación estándar de 11.02.

La variable independiente de interés del segundo modelo es la desviación con respecto a la media de proporciones obtenida en la categoría nacional que ocupó el primer lugar en la boleta en los distritos donde se registró integración cooperativa en la elección anterior. La misma da a lugar a una variable continua donde los resultados positivos son interpretados como una satisfacción de los candidatos nacionales respecto al desempeño de sus agentes a nivel de distrito, la cual los incentivaría a volver a delegar en ellos en los próximos comicios. Por su parte, los distritos que arrojan resultados negativos indican la situación inversa, a partir de la cual se incrementarían las probabilidades de que el oficialismo nacional adopte estrategias que fomenten la competitividad entre agentes alternativos en dichos municipios. En sus 106 observaciones, esta variable presenta una media de cero, unos valores máximos y mínimos de .17 y -.26 junto con una desviación estándar de .07 .

Dado que en las dos primeras consecuencias observacionales de la segunda hipótesis se testea la relevancia de las estrategias pretéritas de intendentes y presidentes en las decisiones de sus contrapartes en los siguientes comicios, las variables de interés de ambos modelos se construyen a partir del rezago de las variables dependientes del primer y segundo modelo. En este sentido, la variable independiente del tercer modelo es el tipo de integración ascendente de la última elección, mientras que en el cuarto este lugar le corresponde a la estrategia descendente de los últimos comicios.

6 La categoría nacional que ocupa el primer lugar en la boleta corresponde al cargo nacional de mayor relevancia que se pone en disputa en dicha elección. En los comicios ejecutivos este lugar es ocupado por los candidatos a la presidencia, mientras que en los comicios legislativos corresponde a diputados o senadores nacionales. Esta aclaración también vale para la variable independiente del segundo modelo y para la variable de control del número efectivo de partidos. 
$\mathrm{Al}$ igual que las variables dependientes (y a diferencia de las de interés, ubicadas en t-1), las variables de control corresponden a variables del presente que pueden afectar el resultado de las dependientes de cada uno de los modelos y, de esta forma, presentarán utilidad para reducir la varianza de las relaciones causales (Morgan y Winship, 2007) postuladas hasta el momento. Al respecto, se emplearon tres variables de control: el tipo de elección, la proporción de intendentes que adoptaron estrategias competitivas ascendentes sobre aquellos que se integraron en forma cooperativa y el número efectivo de partidos en la categoría nacional que aparece en la boleta en primer lugar.

La primera de ellas, distingue en forma dicotómica si en los comicios se eligen cargos ejecutivos o si sólo se renuevan bancas legislativas, asignándose el valor de 1 en el primer caso y de 0 en el segundo. Su inclusión en los cuatro primeros modelos se justifica por los ciclos de auge y declive que pueden atravesar los partidos de gobierno que los lleva a cosechar mejores performances en elecciones ejecutivas en comparación con las de medio término. Esta dinámica, de la cual no estuvo exento el FPV (si se exceptúan las elecciones de 2005), fue explorada originalmente por literatura estadounidense (Alesina y Rosenthal, 1995), siendo replicada en otros contextos (Kern y Hainmuller, 2006). La misma concibe a las elecciones de medio término como comicios moderadores, donde un sector del electorado tiene la oportunidad de fomentar un balance horizontal (Kedar, 2006; Gaines y Crombez, 2004) del poder del presidente votando a partidos de oposición para el Congreso.

Contrariamente a lo que sucede en los comicios ejecutivos concurrentes, donde los candidatos nacionales con perspectivas de triunfo pueden llevar a posiciones de gobierno a postulantes de su partido que ubican en su boleta (Campbel, 1991), en el marco de elecciones legislativas concurrentes, donde se emplea un método de votación de boleta y sobre que dificulta la división del voto (Barnes et. al, 2017), los intendentes del conurbano pueden anticipar las desventajas afrontar los comicios de renovación de sus Concejos Deliberantes en representación del oficialismo nacional.

A partir de lo señalado, cabría esperar que en los casos de elecciones legislativas se incrementen las posibilidades de integración competitiva ascendente respecto a los comicios ejecutivos, debido a las (des)ventajas que representa para los intendentes peronistas del conurbano competir con la etiqueta del oficialismo nacional en cada caso. Por contrapartida, se espera que las posibilidades de implementar estrategias competitivas descendentes se incrementen en el marco de elecciones ejecutivas en relación con las legislativas, en tanto que en este último tipo de elección, las estrategias competitivas descendentes podrían poner en duda la cooperación de los intendentes, 
dados los escasos incentivos de coordinación ascendentes que presentan los comicios de medio término.

En estricta relación con lo anterior, se incorpora al segundo y tercer modelo otra variable de control: la proporción de intendentes que adoptaron estrategias competitivas ascendentes sobre aquellos que se integraron en forma cooperativa, la cual se obtiene dividiendo la cantidad de jefes municipales que adoptó cada una de las estrategias en la misma elección. Esta operación da lugar a una variable continua cuyos valores se extienden de cero (en casos como las elecciones de 2007 y 2009, cuando ningún intendente del conurbano adoptó estrategias competitivas) hasta 1.4 (correspondiente a los comicios de 2005, cuando 10 intendentes apoyaron la candidatura a Senadora Nacional de Hilda Duhalde). En este caso se espera que los incrementos en esta variable disminuyan la probabilidad de los candidatos nacionales de adoptar estrategias competitivas descendentes, ya que una mayor cantidad de intendentes que declaran sus estrategias competitivas podría disuadir a los (candidatos de los) presidentes a estimular la competitividad en los distritos restantes, donde sus jefes municipales adoptan estrategias cooperativas ante la posibilidad de que estos últimos cambien su estrategia antes del «cierre de listas».

La variable de control restante es el número efectivo de partidos en la categoría nacional, la cual aparece en la boleta en primer lugar en cada uno de los distritos analizados. Esta se incluye en el primer y cuarto modelo por su relevancia en elecciones de cargos en múltiples arenas en elecciones concurrentes, en tanto la congruencia entre los sistemas partidarios a nivel nacional y municipal se incrementa a medida que disminuye el número efectivo de candidaturas nacionales (Borges y Lloyd, 2016). En tanto se carece de los datos relativos a la cantidad efectiva de candidatos nacionales previa a los procesos electorales, se empleará como proxy el número efectivo de partidos registrado en dichas categorías, con la expectativa de que los incrementos unitarios de esta variable continua (que presentó una media de 3.21, desviación estándar de .51 y un rango que se extendió entre 1.7 y 4.4) aumenten las probabilidades de integración competitiva de los intendentes del conurbano.

Es preciso señalar que la inclusión de este proxy se enfrenta con una limitación, en tanto se sirve de un indicador obtenido con posterioridad a las elecciones (como la fragmentación del voto) para controlar una estrategia de integración ascendente que se implementa antes de los comicios. Asimismo, debido al efecto arrastre invertido (Ames, 1994; Bechtel, 2012) una estrategia de coordinación o competición implementada por un intendente posee una influencia relevante en el resultado de las categorías nacionales en su municipio, este indicador podría estar expuesto a sesgos. Más 
allá de lo señalado, es preciso considerar que, al igual que sucede con la capacidad de anticipar escenarios propios de elecciones legislativas o ejecutivas, los incumbents municipales poseen la posibilidad de prever (al menos en forma estimada) la fragmentación del voto en las categorías nacionales de los próximos comicios en momentos previos al «cierre listas» y, en función de ello, de definir su estrategia de integración multinivel.

\section{Resultados}

Este apartado tiene por objeto exhibir el testeo de las dos hipótesis presentadas. Para ello, se aborda cada una de sus consecuencias observacionales por separado en el orden en que fueron presentadas, destacando las conclusiones más relevantes que se derivan de los resultados obtenidos.

\section{TABLA 1}

Efecto de la diferencia registrada entre la proporción de votos obtenidos en las categorías municipales y nacionales sobre las probabilidades de integración competitiva ascendente en la próxima elección en 24 distritos del conurbano bonaerense (2005-2015)

\begin{tabular}{|l|c|}
\hline Diferencia de proporciones de votos entre categorías municipales y nacionales & $1.084^{* * *}$ \\
\hline & {$[0.028]$} \\
\hline Elección de cargos ejecutivos & $0.348^{*}$ \\
\hline & {$[0.193]$} \\
\hline Número efectivo de partidos en la categoría nacional & $2.37^{*}$ \\
\hline Constante & {$[1.138]$} \\
\hline & $0.0183^{* *}$ \\
\hline Número de observaciones & {$[0.030]$} \\
\hline Número de distritos & 122 \\
\hline Pseudo $\mathrm{R}^{2}$ & 24 \\
\hline
\end{tabular}

* significativo al $10 \%, * *$ al $5 \%, * * *$ al $1 \%$

Fuente: Elaboración propia.

7 De un total de 144 casos potenciales que presenta el estudio de 6 elecciones en los 24 distritos del conurbano, se han tenido en cuenta para el primer modelo 122 casos. La omisión de las 22 observaciones restantes responde a que no fueron contemplados aquellos municipios donde el intendente no compitió en la boleta de los candidatos nacionales del peronismo, o bien, los primeros casos donde flamantes intendentes peronistas compitieron en elecciones, por carecer de datos de la elección anterior.

De los 122 casos analizados, $22(19.04 \%)$ presentaron integraciones competitivas, mientras que los 100 restantes $(81.96 \%)$, constituyeron casos de cooperación multinivel. 
En lo que respecta al primer enunciado observacional de la primera hipótesis, la diferencia de votos registrada entre las categorías municipales y nacionales posee un efecto positivo esperado y significativo al $1 \%$ sobre la estrategia a implementar por parte de los intendentes en la elección posterior. El coeficiente arrojado por el modelo da cuenta de que un incremento en una unidad en el valor registrado por la variable de diferencia, incrementa en promedio un $8 \%$ la razón de momios de probabilidad de que se registre una integración competitiva en la siguiente elección, descontando el efecto del tipo de elección y la cantidad efectiva de partidos nacionales en cada distrito.

El sentido de los coeficientes de las variables de control también presenta la dirección esperada por la teoría, evidenciando ambos un nivel de significancia del $10 \%$. Acorde al resultado de los coeficientes, las elecciones donde se eligen cargos ejecutivos disminuyen en promedio un $66 \%$ la razón de momios de probabilidad de integración competitiva, mientras que un incremento unitario en el número efectivo de partidos nacional al interior de un distrito incrementa esta razón de probabilidades en un $137 \%$.

Como se observa en los resultados del segundo modelo, la desviación con respecto a la media de los registros obtenidos en las categorías nacionales entre los intendentes que adoptan estrategias de integración cooperativa posee una incidencia significativa en la competitividad que estos deben afrontar en los próximos comicios. Acorde al coeficiente que plantea nuestra variable de interés, cada incremento unitario de la misma redunda en una disminución promedio de doce desviaciones estándar en la variable dependiente. En este sentido, si se multiplica este coeficiente por la desviación estándar de la variable de interés (0.07), es posible señalar que cada incremento en una desviación estándar de la media de votos obtenida en la elección anterior, reduce en 0.943 la competitividad al interior del distrito en los comicios siguientes.

Por su parte, las variables de control resultan significativas al 5\% y marcan, por un lado, un incremento promedio de algo más de media desviación estándar (.59) respecto a la media de competitividad cuando se eligen cargos ejecutivos y, por otra parte, una disminución promedio superior a media desviación estándar (-.63) en relación a la media registrada en la competitividad en situaciones donde se incrementa la proporción de intendentes que adoptan estrategias competitivas en la misma elección. 


\section{TABLA 2}

Efecto de los resultados categorías nacionales en la elección anterior sobre el nivel de competitividad descendente registrado al interior de los 24 distritos del conurbano bonaerense (2005-2015)

\begin{tabular}{|l|c|}
\hline $\begin{array}{l}\text { Desviación de la proporción de votos en categorías nacionales obtenidas } \\
\text { en un distrito con respecto a la media de distritos que adoptaron } \\
\text { estrategias cooperativas en la elección anterior }\end{array}$ & $-12.85^{* *}$ \\
\hline & {$[3.03]$} \\
\hline Elección de cargos ejecutivos & $0.59^{*}$ \\
\hline & {$[0.24]$} \\
\hline Proporción de intendentes que adoptan estrategias competitivas & $-0.63^{*}$ \\
\hline & {$[0.25]$} \\
\hline Constante & -0.20 \\
\hline & {$[0.17]$} \\
\hline Número de observaciones & 8 \\
\hline Número de distritos & 106 \\
\hline $\mathrm{R}^{2}$ within & 24 \\
\hline $\mathrm{R}^{2}$ between & 0.18 \\
\hline $\mathrm{R}^{2}$ overall & 0.15 \\
\hline
\end{tabular}

$*$ significativo al $.05 \%, * *$ al $.001 \%$

Fuente: Elaboración propia.

8 Para el segundo modelo el número de observaciones se redujo a 106, es decir, 18 menos que los casos empleados para testear la primera hipótesis. Esto se debe a que fueron omitidos los 22 casos de integración competitiva contemplados en el modelo anterior. La omisión se justifica en el énfasis en los procesos de integración multinivel entre oficialismos de distintos niveles de competencia, razón por la cual solo se analizó en la variación de las estrategias de integración descendentes del oficialismo nacional en los casos donde los intendentes también adoptaron estrategias cooperativas, ya que cuando estos compitieron en otras boletas, la competitividad al interior del FPV en sus distritos no fue el resultado de una negociación multinivel entre oficialismos.

Cabe señalar que, más allá de estas omisiones, las características de nuestras variables independientes permitieron la incorporación de los casos correspondientes a la de primera integración cooperativa de los municipios de Tigre, General San Martín, Esteban Echeverría, San Isidro y Morón, que no habían podido ser analizados en el primer modelo. 


\section{TABLA 3}

Efecto del tipo de integración implementado en la elección anterior sobre el nivel de competitividad descendente afrontado por los intendentes del conurbano bonaerense en las elecciones siguientes (2005-2015)

\begin{tabular}{|l|c|}
\hline Integración competitiva del intendente en la elección anterior & $1.82^{* *}$ \\
\hline Elección de cargos ejecutivos & {$[0.44]$} \\
\hline & 0.17 \\
\hline Proporción de intendentes que adoptan estrategias competitivas & {$[0.74]$} \\
\hline & -0.24 \\
\hline Constante & {$[0.17]$} \\
\hline & $-0.30^{*}$ \\
\hline Número de observaciones ${ }^{9}$ & {$[0.14]$} \\
\hline Número de distritos & 102 \\
\hline $\mathrm{R}^{2}$ within & 23 \\
\hline $\mathrm{R}^{2}$ between & 0.23 \\
\hline $\mathrm{R}^{2}$ overall & 0.14 \\
\hline
\end{tabular}

* significativo al $5 \%, * *$ al $.01 \%$

Fuente: Elaboración propia.

Los resultados expuestos en la Tabla 3 indican que los oficialismos nacionales tienden a emular las estrategias precedentes de los alcaldes municipales en comicios sucesivos tal como postula la primera consecuencia observacional de la segunda hipótesis. En función de los coeficientes de nuestra variable de interés, es posible afirmar que cuando algún intendente del conurbano adoptó una estrategia competitiva en la elección anterior y dos años más tarde decidió competir dentro del FPV, este afrontó en su distrito una competitividad promedio que se incrementó en 1.82 desviaciones estándar con respecto a la media. Si se procede igual que en el modelo anterior y se multiplica este coeficiente con la desviación estándar de la variable de interés, podemos concluir que la competitividad descendente se incrementó en promedio 0.12 en los distritos donde los intendentes adoptaron una estrategia de competición ascendente en los comicios previos.

9 Cabe señalar que, en comparación con el segundo modelo, se han omitido los casos correspondientes a los intendentes que se integraron con los oficialismos nacionales por primera vez en las elecciones de 2007 (General San Martín, Esteban Echeverría y San Isidro) y 2011 (Morón), debido a la imposibilidad de asignarle un valor a la variable de interés en estos casos. 
Cabe señalar que el sentido negativo que arroja el coeficiente de la constante y su nivel de significancia al 5\%, nos permiten afirmar que en el marco de elecciones de renovación legislativa donde ningún jefe municipal del conurbano adopta estrategias competitivas, los intendentes que se integraron en forma cooperativa en la elección anterior enfrentan en sus distritos una competitividad que se reduce en promedio .30 desviaciones estándar con respecto a la media, resultado que también es coherente con la hipótesis presentada.

Antes de exponer los resultados de cuarto modelo, cabe señalar que el rezago de la variable de competitividad redujo la cantidad de observaciones disponibles a un total de 84 , debido a que no se han podido incorporar los casos de los intendentes que adoptaron estrategias de integración competitivas en las elecciones precedentes, ni se han podido contemplar los datos del comicio previo de aquellos intendentes que se integraron por primera vez a los candidatos nacionales del FPV. La consecuencia más significativa de la reducción de las observaciones disponibles ha sido reducir los «casos de éxito» de nuestra variable dependiente, es decir, las situaciones donde los intendentes se integraron en forma competitiva en las siguientes elecciones a sólo 6 (lo cual equivale al 7\% de las 84 observaciones contempladas).

\section{TABLA 4}

Efecto de la competitividad descendente registrada en la elección anterior sobre el tipo de integración ascendente implementada en los próximos comicios en los distritos del conurbano bonaerense (2005-2015)

\begin{tabular}{|l|c|}
\hline Competitividad descendente en la elección anterior & 1.59 \\
\hline & {$[0.91]$} \\
\hline Elección de cargos ejecutivos & $0.0033^{*}$ \\
\hline & {$[0.008]$} \\
\hline Número efectivo de partidos en la categoría nacional & $0.0022^{*}$ \\
\hline Constante & {$[0.005]$} \\
\hline & $0.00^{*}$ \\
\hline Número de observaciones & {$[0.00]$} \\
\hline Número de distritos & 84 \\
\hline Pseudo $\mathrm{R}^{2}$ & 23 \\
\hline
\end{tabular}

* significativo al .05\%

Fuente: Elaboración propia. 
Como se evidencia en los resultados presentados, si bien la variable de interés posee un sentido positivo esperado por el marco analítico, la misma no presenta los niveles de significancia necesarios para obtener un testeo exitoso de la segunda consecuencia observacional de la segunda hipótesis. En este punto deben remarcarse las limitaciones referidas al indicador de competitividad como elemento para medir las estrategias descendentes, que ya han sido señaladas en el apartado metodológico.

Para finalizar, el Gráfico 1 presenta un diagrama de dispersión con una línea de regresión suavizada que permite testear la tercera consecuencia observacional de la segunda hipótesis, empleando a cada uno de los 24 distritos analizados como unidad de análisis.

\section{GRÁFICO 1}

Relación entre la mediana de la competitividad y la cantidad de estrategias de integración cooperativas ascendentes en los 24 municipios del conurbano bonaerense (2005-2015)

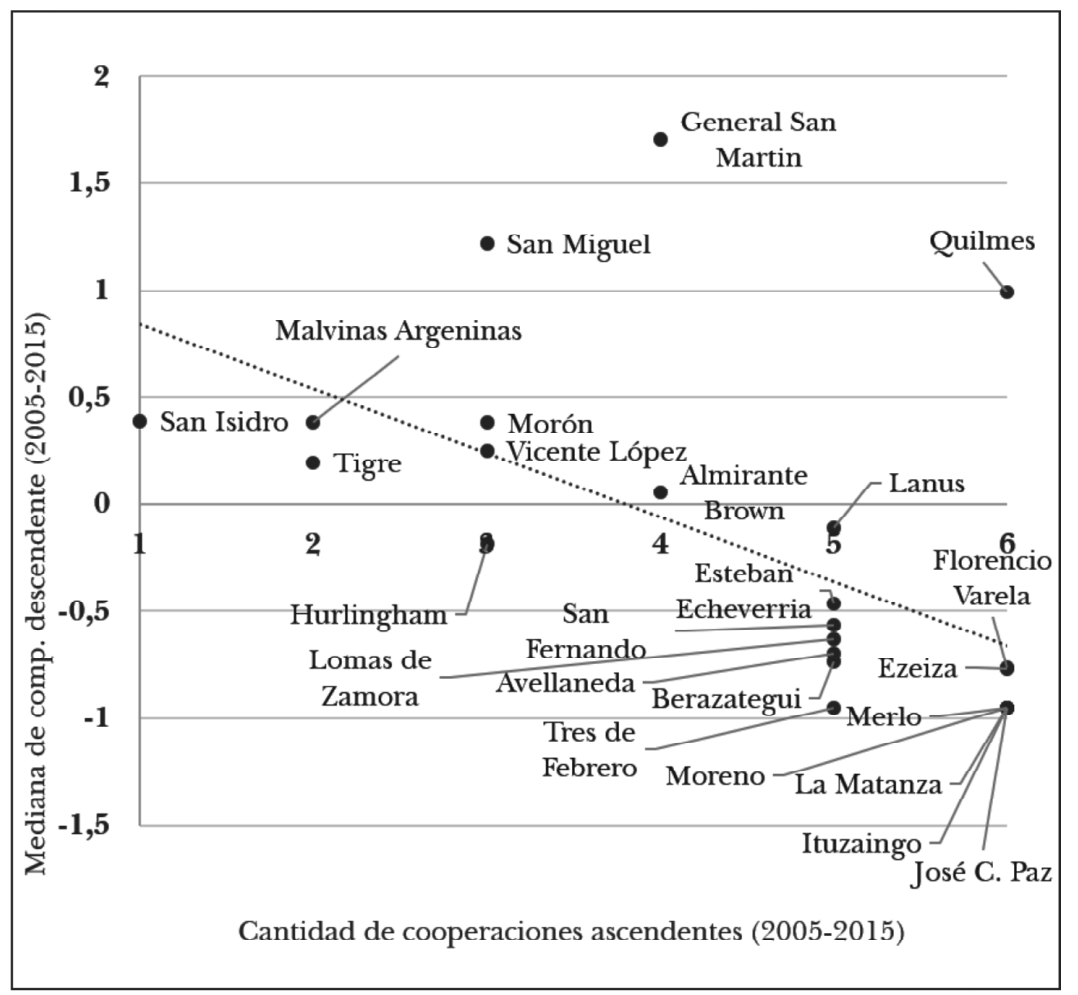

Fuente: Elaboración propia. 
La correlación negativa entre la mediana que presentó competitividad en cada uno de los municipios del conurbano y la cantidad de ocasiones en que sus intendentes implementaron estrategias de cooperación ascendentes (la cual presenta un coeficiente de Pearson de -0.60 con un nivel de significancia de .001), resulta indicativa de la existencia de reciprocidad entre las estrategias de integración adoptadas por los ejecutivos nacionales y municipales al interior de los distritos analizados. Si bien esta correlación no constituye evidencia suficiente de causalidad, la misma ofrece indicios de que tanto oficialismos nacionales como municipales replicaron las estrategias de integración empleadas por sus contrapartes durante las elecciones analizadas a partir de una dinámica que presenta alguna similitud con la de tit for tat.

A su vez, la tendencia apuntada nos permite señalar la existencia de procesos de integración descentralizados al interior del peronismo del conurbano bonaerense, donde se concretaron equilibrios cooperativos en los casos ubicados en el cuadrante inferior derecho del diagrama (Merlo, Moreno, La Matanza, José C. Paz, Ezeiza, Tres de Febrero, Avellaneda, Berazategui, San Fernando y Esteban Echeverría), contextos estos donde presidentes e intendentes implementaron estrategias de cooperación multinivel durante una década. En contraposición, los casos ubicados en la parte izquierda del diagrama, presentan ejemplos de los equilibrios competitivos registrados en los municipios restantes ${ }^{10}$.

\section{Discusión}

Hace casi dos décadas Steven Levitsky (2001) escribió un ensayo titulado «Adentro de la caja negra: estudios recientes de las organizaciones partidarias en América Latina». En el mismo realizaba un balance de la literatura existente e instaba a profundizar los estudios sobre los partidos en la región, cuya estructura interna suele ser concebida como una trama oscura e inaccesible.

Este estudio constituye un intento de explicar algunas características de «la caja negra» que representa para los observadores argentinos las negociaciones pre-electorales del peronismo, particularmente en un ámbito electoralmente determinante como el del conurbano bonaerense. Para ello, se recurrió a dos marcos analíticos que no son habitualmente empleados para el estudio de los

10 En este punto, cabe señalar que los outliers de Gráfico 1 (sobre todo los casos de Quilmes y General San Martín) que presentan alto niveles de competitividad a pesar de haber implementado estrategias de cooperación ascendente, podrían ser indicativos de que la fragmentación electoral al interior de los oficialismos municipales se debió a aspectos locales y no estuvo necesariamente patrocinada por dirigentes nacionales.

Finalmente, cabe mencionar que otra limitación del empleo de distritos como unidad de análisis consiste en la imposibilidad de contemplar recambios en los incumbents municipales en los casos del conurbano donde estos se produjeron. 
partidos políticos: el enfoque de agente-principal y las contribuciones sobre la cooperación que presenta la teoría de juegos. Ambos nos permitieron señalar que la elección anterior impacta sobre la elección siguiente, ya sea por el monitoreo de los resultados electorales o por la reciprocidad respecto a las estrategias pretéritas de oficialismos que compiten en otros niveles.

A diferencia de la propuesta de Levitsky, este trabajo no se enfocó en los partidos políticos como organizaciones, sino como instituciones que resultan del equilibrio entre actores estratégicos (Shepsle, 1989; Crawford y Ostrom, 1995; Leiras, 2006) con un énfasis particular en los incumbents (Lacher, 2018). Este enfoque resulta particularmente útil para analizar la dinámica de competición electoral de partidos estratárquicos, donde la cooperación entre oficialismos no se encuentra garantizada por una autoridad común. En este marco, la posibilidad de que un partido como el peronismo continúe siendo electoralmente exitoso en ámbitos como el conurbano bonaerense descansa en la construcción de equilibrios cooperativos entre actores que compiten en diferentes arenas.

Sin embargo, esta empresa puede frustrarse ante la posibilidad latente de implementación de estrategias competitivas, las cuales representan un riesgo constante a las aspiraciones de los oficialismos peronistas de acceder o continuar ejerciendo sus respectivas posiciones de gobierno. Esto último se evidenció con bastante claridad cuando Aníbal Fernández aludió al «fuego amigo», luego de conocerse los resultados de las elecciones generales en provincia de Buenos Aires en 2015. En estos casos, al igual que ocurre con los aviones, las caídas obligan a revisar las cajas negras.

Más allá resultados obtenidos en el caso estudiado, resulta preciso replicar las hipótesis presentadas en otros partidos políticos que compiten en distintas arenas, en la búsqueda de indagar cómo se manifiestan las estrategias competitivas en su seno y si es que las mismas responden a resultados de elecciones anteriores. Por su parte, el análisis de los partidos como instituciones de equilibrio puede tener relevancia a la hora de orientar el estudio de otros tipos de vínculos entre actores partidarios, inclusive aquellos que tienen lugar en un idéntico nivel de competencia. A su vez, la identificación de situaciones donde actores ocupan en forma simultánea el rol de agentes y principales en interacciones que tienen posibilidad de reiterarse en el tiempo, puede ser útil para contribuir a la explicación de la dinámica que subyace a otro tipo de vínculos políticos, donde sus integrantes se encuentran en una relación de delegación y mutua dependencia, sin importar si estos se desarrollan dentro o fuera de los partidos.

De cara a esta potencial agenda de investigación deberán atenderse las limitaciones de los indicadores a los que ha recurrido este trabajo, ya que la búsqueda de mejores herramientas metodológicas permitirá seguir echando luz sobre lo que (hasta ahora) consideramos como «cajas negras». 


\section{Referencias bibliográficas}

Alesina, A. y H. Rosenthal (1995). Partisan Politics, Divided Government, and the Economy. Cambridge: Cambridge University Press.

Avelino, G., C. Biderman y L. Barone (2013). Do Parties Matter? Intra-Party Linkages and Electoral Performance in Brazil. En Dados, 55, 987-1013.

Axelrod, R. (1984). The Evolution of Cooperation. Nueva York: Basic Books.

Barnes, T., C. Tchintian y S. Alles (2017). Ballot Structure and Split Ticket Voting: Evidence from a Quasi-Experiment. En The Journal of Politics, 79(2), 439-456.

Bendor, J., A. Glazer y T. Hammond (2001). Theories of Delegation. En Annual Review of Political Science, 4(1), 235-269.

Bechtel, M. (2012). Not Always Second order: Subnational Elections, National-level Vote Intentions, and Volatility Spillovers in a Multi-level Electoral System. En Electoral studies, 31(1), 170-183.

Borges, A. y R. Lloyd (2016). Presidential Coattails and Electoral Coordination in Multilevel Elections: Comparative essons from Brazil. En Electoral Studies, 43, 104-114.

Calvo, E. y M. Escolar (2005). La nueva política de partidos en la Argentina: crisis política, realineamientos partidarios y reforma electoral. Buenos Aires: Pent.

Campbell, J. (1991). The Presidential Surge and its Midterm Decline in Congressional Elections». En Journal of Politics, 53, 477-87.

Carty, Kenneth. 2004. Parties as Franchise Systems: The Stratarchical Organizational Imperative. En Party Politics, 10(1), 5-24.

Clerici, P. (2014). Aliados y Contendientes. Dimensionando el Fenómeno de la Congruencia Aliancista en Argentina (1983-2013). [Tesis de Doctorado en Ciencia Política y Estudios Internacionales, Universidad Torcuato Di Tella, Buenos Aires, Argentina]

Clerici, P. (2015). La Congruencia Aliancista de los Partidos Argentinos en Elecciones Concurrentes (1983-2011). En Estudios Políticos, 36, 143-170.

Clerici, P., F. Cruz y L. Goyburu (2016). ¿Los hermanos sean unidos? Competitividad en las primarias municipales en la Provincia de Buenos Aires». En Revista SAAP, 10(2), 279-309.

Crawford, S. y E. Ostrom (1995). A Grammar of Institutions. En American Political Science Review, 89 (3), 582-600.

Downs, A. (1957). An Economic Theory of Political Action in a Democracy. En Journal of Political Economy, 65(2), 135-150.

Gaines B. y C. Crombez (2004). Another Look at Connections Across German Elections. En Journal of Theoretical Politics, 16 (3), 289-319

Galván, F. (2011). ¿El sello importa? El rol partidario de los ejecutivos nacionales y provinciales a la luz de las listas peronistas disidentes (1989-2009). En Revista Postdata, 16 (1), 63-84. 
Galván, F. (2011b). Rupturistas y disidentes. Un estudio sobre las estrategias intrapartidarias en las facciones del PJ y la UCR (1983-2007). Presentado en X Congreso de la Sociedad Argentina de Análisis Político, Córdoba, Argentina.

Kim, M. (2015). Parties without Brand Names: the Causes and Consequences of Party Relabeling. [Tesis de Doctorado, Universidad de Iowa, Estados Unidos]

Kitschelt, H. (2000). Linkages between Citizens and Politicians in Democratic Politics. En Comparative Political Studies, 33, 845-79.

Kedar, O. (2006). How Voters Work around Institutions: Policy Balancing in Staggered Elections. En Electoral Studies, 25 (3), 509-527.

Kern, H. y J. Hainmüller (2006). Electoral Balancing, Divided Government, and Midterm Loss in German State Elections. En Journal of Legislative Studies, 12 (2), $127-149$.

Laakso, M. y R. Taagepera (1979). «Effective» Number of Parties: A Measure with Application to West Europe. En Comparative Political Studies, 12 (1), 3-27.

Lacher, A. (2018). Estrategias de integración multinivel desde un enfoque de agente-principal: el caso del peronismo del conurbano bonaerense (2005-2015). [Tesis de Maestría, FLACSO, Ciudad de México, México]

Leiras, M. (2006). Parties, Provinces and Electoral Coordination: a Study on the Determinants of Party and Party System Aggregation in Argentina (1983-2005). [Tesis de Doctorado, Universidad de Notre Dame, Indiana, Estados Unidos].

Levitsky, S. (2001). Inside the Black Box: Recent Studies of Latin American Party Organizations. En Studies in Comparative International Development, 36 (2), 92-110.

Lodola, G. (2009). La estructura subnacional de las carreras políticas en Argentina y Brasil. En Desarrollo Económico, 49 (194), pp. 247-286.

Lupia, A. y M. McCubbins (2000). Representation or Abdication? How Citizens Use Institutions to Help Delegation Succeed. En European Journal of Political Research, 37, 291-307.

Mauro, S. (2018). Coalition Politics in a Federalized Party System: The Case of Argentina en Albala, A. y Reniu, J (Eds.), Coalition Politics and Federalism. Springer.

Moe, T. (1984). The New Economics of Organization. En American Journal of Political Science, 28 (4), 739-777.

Morgan, S. L., y Winship, C. (2007). Counterfactuals and Causal Inference: Methods and Principles for Social Research. Nueva York: Cambridge University Press.

Ollier, M. (2010). El liderazgo político en democracias de baja institucionalización (el caso del peronismo en la Argentina). En Revista de Sociología, 24, 127-150.

Samuels, D. (2003). Ambition, Federalism, and Legislative Politics in Brazil. Cambridge: Cambrige University Press.

Scherlis, G. (2013). Presidentes y partidos en América Latina: la excepcionalidad del peronismo en el contexto latinoamericano. En Politai: Revista de Ciencia Política. $4(7), 29-50$. 
Shepsle, K. (1989). Studying Institutions: Some Lessons from the Rational Choice Approach. En Journal of Theoretical Politics, 1 (2): 131-147.

Szwarcberg Daby, M. (2016). Reelecciones infinitas: el caso de los intendentes del Conurbano. En Revista POSTData, 21(2), 577-592.

Van Houten, P. (2009). Multi-level Relations in Political Parties: A Delegation Approach. En Party Politics, 15(2), 137-156.

\title{
Palabras Clave
}

integración multinivel — nivel municipal — principal-agente — cooperación - partidos políticos

\begin{abstract}
The decentralized character of multilevel integration in stratarchical parties that compete in different arenas, becomes evident in the complex pre-electoral negotiations that take place between incumbents. As a result, the national, provincial and municipal incumbents can adopt cooperation and competition strategies that impact on the competence arenas of their counterparts. The goal of this paper is to explain the variation of these strategies between presidents and mayors of the 24 municipalities of the Buenos Aires' suburbs in the argentinian peronism, from the analysis of electoral results and integration strategies corresponding to the previous elections. In this vane, the link between incumbents of different arenas will be conceptualized as a relationship between principals and agents, while it will be resorted a cooperation approach. The observable consequences of this analytical frame give rise to two hypotheses, which observational consequences are tested through the employment of logistics regressions and fixed effects panels.
\end{abstract}

\section{Keywords}

multilevel integration - municipal level — principal-agent — cooperation political parties 